\title{
Rothmund-Thomson Syndrome: A 13-Year Follow-Up
}

\author{
Guillermo Antonio Guerrero-González ${ }^{\mathrm{a}} \quad$ Sylvia Aideé Martínez-Cabriales ${ }^{\mathrm{a}}$ \\ Aideé Alejandra Hernández-Juárez ${ }^{b}$ José de Jesús Lugo-Trampe ${ }^{b}$ \\ Nelly Alejandra Espinoza-González ${ }^{\mathrm{a}} \quad$ Minerva Gómez-Flores ${ }^{\mathrm{a}}$ \\ Jorge Ocampo-Candiani ${ }^{\mathrm{a}}$ \\ Departments of ${ }^{\mathrm{a}}$ Dermatology and ${ }^{\mathrm{b}}$ Genetics, Hospital Universitario 'Dr. José Eleuterio \\ González', Universidad Autónoma de Nuevo León, Monterrey, Mexico
}

\section{Key Words}

Rothmund-Thomson syndrome · Poikiloderma - Congenital bone defects · Malignancy · Osteosarcoma $\cdot$ RECQL4 · Follow-up

\begin{abstract}
Rothmund-Thomson syndrome (RTS) is a rare autosomal recessive disorder presenting with poikiloderma and other clinical features, affecting the bones and eyes and, in type II RTS, presenting an increased risk for malignancy. With about 300 cases reported so far, we present a 13-year follow-up including clinical images, $\mathrm{X}$-rays and genetic analysis. A 13-monthold female started with a facial rash with blisters on her cheeks and limbs at the age of 3 months along with congenital hypoplastic thumbs, frontal bossing and fine hair, eyebrows and eyelashes. The patient was lost to follow-up and returned 12 years later with palmoplantar hyperkeratotic lesions, short stature, disseminated poikiloderma and sparse scalp hair, with absence of eyelashes and eyebrows. Radiographic analysis showed radial ray defect, absence of the thumb and three wrist carpal bones, and reduced bone density. Gene sequencing for the RECQL4 helicase gene revealed a mutation on each allele. RTS is a rare disease, and in this patient we observed the evolution of her skin lesions and other clinical features, which were important for the classification of type II RTS. The next years will provide even more information on this rare disease.


Guerrero-González et al.: Rothmund-Thomson Syndrome: A 13-Year Follow-Up

\section{Introduction}

Rothmund-Thomson syndrome (RTS) is a rare autosomal recessive disorder. To date around 300 cases have been reported and two clinical forms have been described: type I, characterized by poikiloderma, ectodermal dysplasia and juvenile cataracts with unknown etiology, and type II with poikiloderma, congenital bone defects, an increased frequency of malignancy (specially osteosarcoma) and RECQL4 (8q24.3), the only gene whose mutations are known to cause RTS. It has yet to be clarified whether these two forms represent two different syndromes with common features or clinical entities involving different genes acting on the same pathway [1].

\section{Case Report}

We present a 13-month-old female with facial rash and blisters on the cheeks and limbs starting at the age of 3 months along with congenital hypoplastic thumbs, frontal bossing and fine hair, with thinning of brows and eyelashes. Skin biopsy showed epidermal atrophy with dermoepidermoid edema, and RTS was diagnosed (fig. 1). The patient returned 13 years later with short stature, sparse scalp hair, absence of eyelashes and brows, disseminated poikiloderma, café au lait spots on the trunk and palmoplantar hyperkeratotic lesions. Radiographic analysis showed radial ray defect, absence of the thumb and three wrist carpal bones, and reduced bone density (fig. 2). No other alterations were found. Karyotype analysis was normal $(46, \mathrm{XX})$. Gene sequencing revealed mutations in both alleles of the RECQL4 helicase gene. One of them is a nonsense recurrent mutation located in exon 14, consisting of a change of a cytosine for a thymine (c.2269C $>$ T) producing a premature stop codon and therefore a truncated protein (p.Q757X). On the other allele, the mutation observed has been described in few reports in a compound heterozygous state with c. $2269 \mathrm{C}>\mathrm{T}$. This mutation is a deletion of two nucleotides found in exon 5 (c.1048_1049delAG) which produces a frameshift (p.Arg350Glyfs*21).

\section{Discussion}

RTS is a rare disease and because of its broad clinical spectrum, which includes some common features as well as some individual reports on clinical manifestations and abnormalities, patients can be easily misdiagnosed $[2,3]$. This sheds light on the fact that this is a disease that is far from being completely described and understood. In this patient we observed the evolution of her skin lesions and other clinical features which, along with the mutations of the RECQL4 helicase gene, were important for the classification of type II RTS. In our patient, one of the mutations occurs in the domain of nuclear localization signal (extending from exon 5 through 8). The other mutation is located in the helicase domain encoded by exons 8-14. Both findings are consistent with the literature, since nearly half of the mutations occur before or in the helicase domain, which is considered essential for the proper function of the protein, and represent nonsense, missplicing or frameshift mutations resulting in a truncated protein with impaired helicase activity $[1,4,5]$.

Even though dermatologic screening and radiographic assessment were performed with no evidence of neoplastic disease, continual screening for malignancy is warranted due the high prevalence of osteosarcoma and skin cancer in these patients [1]. The follow-up for the next years will further improve our understanding of the disease. 
Guerrero-González et al.: Rothmund-Thomson Syndrome: A 13-Year Follow-Up

\section{References}

1 Larizza L, Roversi G, Volpi L: Rothmund-Thomson syndrome. Orphanet J Rare Dis 2010;5:2.

-2 Wang LL, Levy ML, Lewis RA, Chintagumpala MM, Lev D, Rogers M, Plon SE: Clinical manifestations in a cohort of 41 Rothmund-Thomson syndrome patients. Am J Med Genet 2001;102:11-17.

-3 Moss C: Rothmund-Thomson syndrome: a report of two patients and a review of the literature. Br J Dermatol 1990;122:821-829.

-4 Siitonen HA, Sotkasiira J, Biervliet M, Benmansour A, Capri Y, Cormier-Daire V, Crandall B, Hannula-Jouppi K, Hennekam R, Herzog D, Keymolen K, Lipsanen-Nyman M, Miny P, Plon SE, Riedl S, Sarkar A, Vargas FR, Verloes A, Wang LL, Kääriäinen H, Kestilä M: The mutation spectrum in RECQL4 diseases. Eur J Hum Genet 2009;17:151-158.

5 Kohzaki M, Chiourea M, Versini G, Adachi N, Takeda S, Gagos S, Halazonetis TD: The helicase domain and C-terminus of human RecQL4 facilitate replication elongation on DNA templates damaged by ionizing radiation. Carcinogenesis 2012;33:1203-1210.

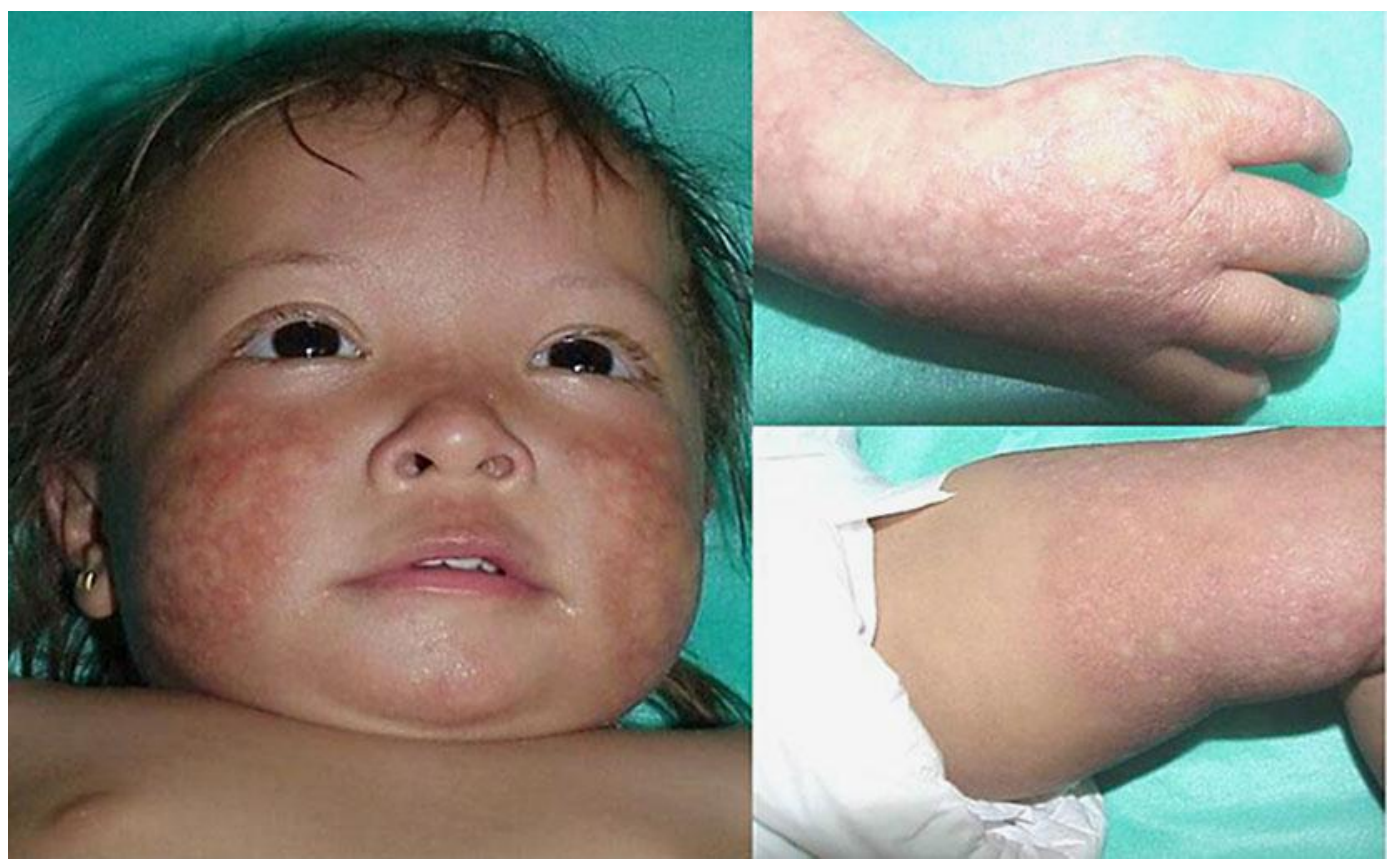

Fig. 1. RTS. Thirteen-month-old patient showing poikiloderma on the face and extremities. 
Guerrero-González et al.: Rothmund-Thomson Syndrome: A 13-Year Follow-Up

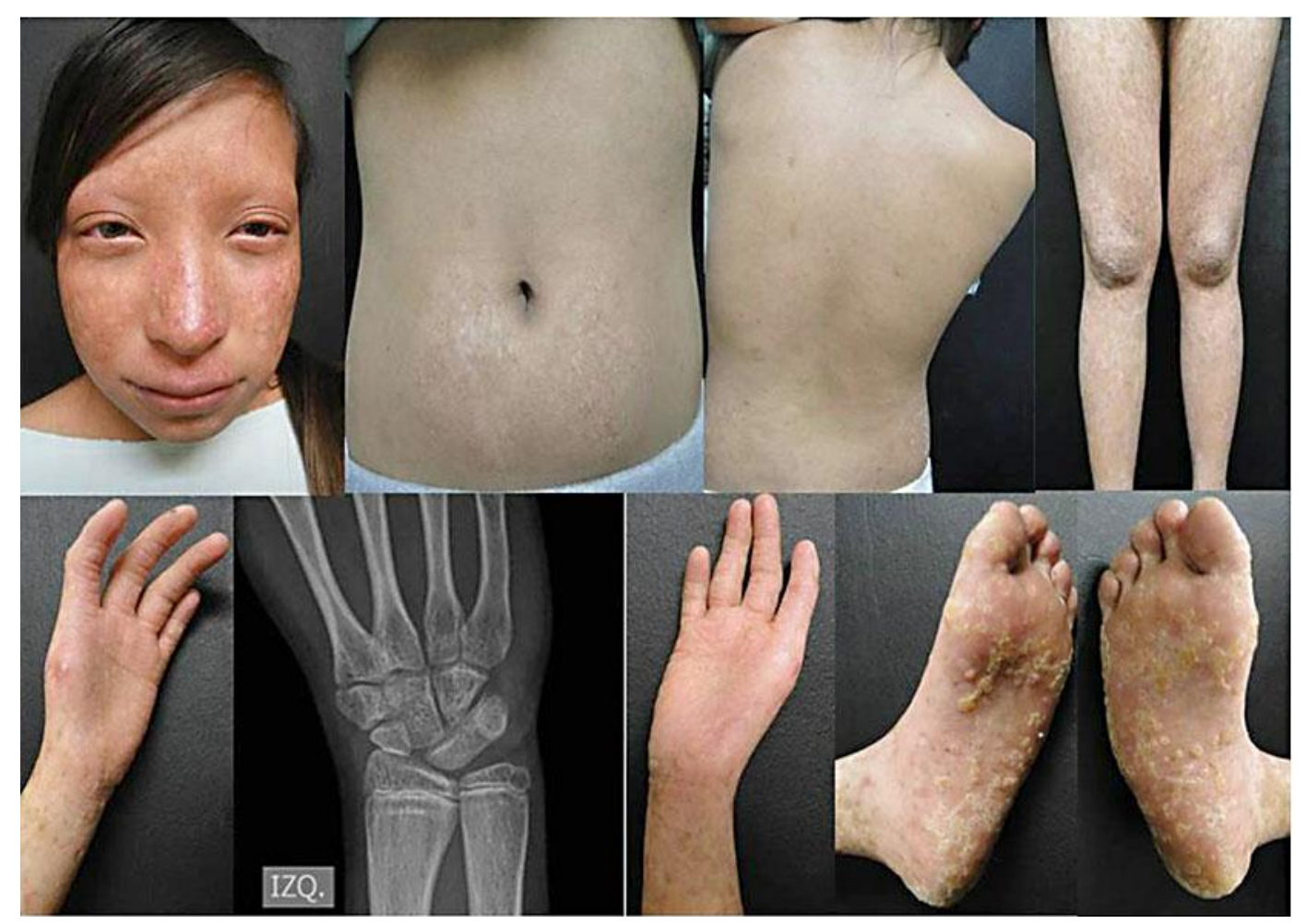

Fig. 2. RTS. Clinical features at the age of 13 years, including bone defects seen on X-ray. 